\title{
Uso de mascarillas faciales como medida de prevención en el contexto de la pandemia por SARS-CoV-2
}

\section{Face masks use as a preventive measure in the context of the SARS-CoV-2 pandemic}

\author{
Victoria Eugenia Montaño-Luna,* María Guadalupe Miranda-Novales ${ }^{\ddagger++}$ \\ * Departamento de Infectología, Hospital de Pediatría; * Unidad de Investigación en Análisis y Síntesis de la \\ Evidencia, Centro Médico Nacional Siglo XXI, Instituto Mexicano del Seguro Social, Ciudad de México.
}

\section{INTRODUCCIÓN}

La actual pandemia por COVID-19 ha afectado a un gran número de personas, reportándose hasta finales de octubre del 2020 más de 37 millones de casos y un millón de muertes en el mundo, en donde casi la mitad de los casos (48\%) y muertes (55\%) se ha notificado en el continente americano. México, Estados Unidos de Norteamérica, Brasil, Argentina y Colombia registraron el número más alto de muertes. ${ }^{1}$

La COVID-19 es una enfermedad respiratoria causada por SARS-CoV-2, un beta-coronavirus de la misma clase de otros coronavirus causantes de las pandemias de SARS y MERS en 2002 y 2012 respectivamente; corresponde a un grupo diverso de virus que infectan diferentes animales y son capaces de causar infecciones respiratorias en humanos. ${ }^{2,3}$

A finales de diciembre del 2019 en Wuhan, en la provincia de Hubei en China, se reportaron los primeros casos de pacientes con neumonía asociados epidemiológicamente a exposición en un mercado de mariscos y animales exóticos. ${ }^{4}$

Después se identificaron otros casos en los que no se documentó historia de exposición al mercado men-

\footnotetext{
+ Correspondencia: MGMN, guadalupe.mirandan@imss.gob.mx Conflicto de intereses: Las autoras declaran que no tienen. Citar como: Montaño-Luna VE, Miranda-Novales MG. Uso de mascarillas faciales como medida de prevención en el contexto de la pandemia por SARS-CoV-2. Rev Mex Pediatr. 2020; 87(5); 163-169. https://dx.doi.org/10.35366/97169
}

cionado, dejando evidencia clara de la transmisión de persona a persona del nuevo virus cuyo genoma fue identificado en enero de 2020. ${ }^{5-8}$ La enfermedad fue nombrada por la Organización Mundial de la Salud como COVID-19 el 11 de febrero del 2020, y un mes después fue declarada una pandemia.

Hasta el momento, se conoce que el espectro clínico y la gravedad de la enfermedad pueden variar desde casos asintomáticos, con enfermedad leve con síntomas que incluyen: fiebre, fatiga, cefalea, diarrea, odinofagia, anorexia y vómito, entre otros; hasta una enfermedad respiratoria grave, que puede evolucionar a falla respiratoria, sepsis, choque y por último disfunción orgánica múltiple y muerte. ${ }^{9-11}$ En niños, el espectro clínico varía, siendo más frecuente un cuadro clínico con síntomas leves, como fiebre y tos, y una gran proporción de pacientes asintomáticos. ${ }^{12,13} \mathrm{El}$ periodo de incubación es de cinco a seis días en promedio, extendiéndose hasta 14 días.

Después de haber analizado múltiples opciones terapéuticas, no existe un tratamiento específico; sin embargo, se siguen realizando investigaciones mediante ensayos clínicos a gran escala para evaluar los diferentes tratamientos propuestos para COVID-19.

\section{TRANSMISIÓN DE SARS-CoV-2}

La propagación del virus ocurre principalmente a través del contacto cercano de persona a persona, (a menos de 1.8 metros). La facilidad con la que el virus se propaga de persona a persona puede variar, es 
más eficaz que el virus de la influenza, pero no tanto como el virus del sarampión. Esta transmisión se ha atribuido parcialmente a la estructura del virus. $\mathrm{Al}$ igual que otros virus respiratorios, SARS-CoV-2 se transmite a través de gotas y contacto directo, existe gran debate acerca de la transmisión aérea. En principio, no hubo duda que el virus se pudiera transmitir durante procedimientos que generan aerosoles (p. ej: intubación endotraqueal, administración de nebulizaciones, aspiración de secreciones), pero este mecanismo de transmisión es menos claro en un entorno comunitario. ${ }^{14,15} \mathrm{La}$ forma más común de transmisión son las gotas expulsadas por una persona al hablar, toser o estornudar, que alcanzan la cara de otra persona durante la exposición cercana. Se ha definido como contacto cercano el que se tiene con una persona infectada a una distancia menor a seis pies (1.8 metros) durante un total acumulativo de 15 minutos o más, en un periodo de 24 horas; que se tomará en cuenta desde dos días previos al inicio de síntomas en la persona infectada. Lo anterior es independiente del uso de equipo de protección. ${ }^{16}$

La transmisión por aerosoles se define como el resultado de la inhalación de partículas menores a $5 \mu \mathrm{m}$. Un aerosol se define como una colección sólida o líquida de partículas suspendidas en un gas, como es el aire y puede contener partículas de cualquier tamaño. ${ }^{17,18}$

En las enfermedades infecciosas transmitidas por aerosoles, los mecanismos principales mediante los cuales se generan aerosoles son la tos y estornudos. ${ }^{19}$ Aún no está bien establecido si la transmisión de SARS-CoV-2 es posible en el contexto de procesos que no generen aerosoles. Existe evidencia de la presencia de ARN viral en estudios realizados con dispositivos automatizados que difícilmente reflejan las condiciones humanas de la tos, pero esto no asegura la viabilidad del virus y, por lo tanto, no podría ser transmitido y desencadenar una infección. ${ }^{20,21}$

La transmisión por gotas se define como la inhalación de partículas mayores a $5 \mu \mathrm{m}$. La infección por SARS-CoV-2, que resulta de la inhalación de gotas, se transmite cuando una persona entra en contacto cercano (menor de 1.8 metros) con una persona infectada y la persona tose o estornuda, generando partículas de gran tamaño o incluso posterior al contacto cara a cara teniendo como puerta de entrada la boca, nariz o conjuntiva.

La transmisión por contacto puede ser de forma directa o indirecta: en la primera, el patógeno es transferido de una persona infectante a una persona susceptible directamente; en la segunda, el patógeno se transporta de una persona a otra a través de un objeto contaminado como una superficie, es decir, a través de fómites en el entorno de una persona infectada. ${ }^{15,18}$

La transmisibilidad del virus depende de la cantidad de virus viable excretada por una persona ya sea al momento de toser, hablar o estornudar, el tipo de contacto y las medidas de seguridad establecidas en el lugar. También está documentada la transmisión del virus de personas infectadas que excretan el virus sin presentar aún sintomatología, llamándose a esto periodo de transmisión presintomático. ${ }^{22,23}$

Se ha postulado que las infecciones asintomáticas podrían corresponder hasta al $80 \%$ de los casos documentados debido a la alta transmisibilidad durante el periodo de síntomas leves o asintomático. ${ }^{24}$

\section{Uso de diferentes tipos de mascarillas faciales (cubrebocas) en la prevención de la propagación de SARS-CoV-2}

Debido a la pandemia actual de la COVID-19 y los métodos de transmisión descritos, se han establecido medidas de prevención a nivel mundial que incluyen el distanciamiento social (mantener distancia $>1.5$ metros entre persona y persona), el aseo frecuente de manos y el uso continuo de mascarillas faciales (comúnmente llamadas cubrebocas), tanto en la comunidad como en el medio hospitalario, como método de prevención y control de la fuente de la enfermedad. Lo anterior se basa en diversos estudios que demuestran la eficacia de diferentes tipos de mascarillas para evitar la propagación de partículas infectantes. Los estudios sobre otros virus respiratorios, como influenza y coronavirus diferentes a SARS-CoV-2, demuestran que el uso de una mascarilla puede evitar la propagación de partículas infectantes de una persona sintomática a otra. ${ }^{25,26}$

Por lo anterior, se realizó una revisión respecto al tipo (de uso médico o no), el material, así como la eficacia de las mismas en la emisión de aerosoles y gotas, lo cual se describe en la Tabla 1.

Mascarillas de uso médico: se han definido como mascarillas quirúrgicas, generalmente planas o plisadas que se ajustan a la cabeza a través de tiras que van alrededor de las orejas, cabeza o ambos. Diseñadas para alta filtración, adecuada respirabilidad y resistencia a la penetración de fluidos. El ajuste es suelto. El nivel de filtración es de gotas de tres micrómetros. 
Tabla 1: Diferencias entre mascarillas quirúrgicas y respiradores.

\begin{tabular}{|c|c|c|}
\hline Característica & Mascarilla quirúrgica & Respiradores N95 \\
\hline Evaluación y aprobación & FDA (U.S. Food and Drug Administration) & NIOSH, Art. 42 CFR Parte 84 \\
\hline Uso & $\begin{array}{l}\text { Resistente a fluidos, protección contra gotas, } \\
\text { salpicaduras de fluidos corporales u otros fluidos } \\
\text { peligrosos }\end{array}$ & $\begin{array}{l}\text { Reduce la exposición a partículas pequeñas como } \\
\text { aerosoles así como gotas }\end{array}$ \\
\hline Ajuste & Suelto & Muy ajustado \\
\hline Prueba de sello & No requiere & $\begin{array}{l}\text { Requiere verificar sellado cada vez que se coloca } \\
\text { para asegurar su adecuado funcionamiento }\end{array}$ \\
\hline Filtración y fuga & $\begin{array}{l}\text { No otorga protección contra partículas pequeñas. } \\
\text { Suele haber fuga en las orillas de la mascarilla en } \\
\text { donde no se encuentra ajustada a la cara }\end{array}$ & $\begin{array}{l}\text { Filtra al menos el } 95 \% \text { de partículas de aerosoles. } \\
\text { Si está adecuadamente ajustada hay mínimo riesgo } \\
\text { de fuga }\end{array}$ \\
\hline $\begin{array}{l}\text { Tiempo recomendado } \\
\text { para su uso }\end{array}$ & $\begin{array}{l}\text { Se puede utilizar por cuatro horas si se mantiene } \\
\text { seca. Debe desecharse y cambiarse frecuentemente }\end{array}$ & $\begin{array}{l}\text { Debe ser desechada posterior al contacto con un } \\
\text { paciente, sobre todo posterior a procedimientos } \\
\text { generadores de aerosoles. Debe desecharse cuan- } \\
\text { do no tenga adecuado sellado, esté dañada, esté } \\
\text { visiblemente sucia o contaminada }\end{array}$ \\
\hline
\end{tabular}

Adaptado de: Centers for Disease Control and Prevention, National Institute for Occupational Safety and Health. Understanding the difference. Available in: https://www.cdc.gov/niosh/np.

- Respiradores: a diferencia de las mascarillas quirúrgicas, tienen mayor nivel de filtración, con la capacidad de filtrar partículas menores a 0.075 micrómetros. De acuerdo a las regulaciones europeas (EN 149), deben filtrar al menos $94 \%$ de las partículas sólidas de cloruro de sodio y gotas de aceite; de acuerdo a las regulaciones estadounidenses (US N95) y de acuerdo a NIOSH 42, deberán filtrar al menos $95 \%$ de las partículas de cloruro de sodio. Deben quedar ajustados a la cara. ${ }^{27-29}$

- Respiradores con válvula: se trata de respiradores que tienen una o varias válvulas de exhalación, estos dispositivos disminuyen la resistencia al momento de la exhalación y hacen más fácil la respiración; sin embargo, permiten que el aire exhalado no filtrado escape a través de un orificio, permitiendo que las gotas excretadas puedan llegar a otras personas. Por tal motivo, este tipo de mascarillas no son adecuadas como medida de protección para evitar la propagación de COVID-19 en la comunidad, y mucho menos en hospitales en los que se requieran ambientes estériles. Los Centros de Control de Enfermedades de Estados Unidos recomiendan que, en caso de no contar con otra opción, se cubra el respirador con válvula, con una mascarilla de uso médico o casero que no interfiera con el sellado del respirador. ${ }^{30-32}$

\section{- Mascarillas de tipo casero o uso no médico:} son aquellas fabricadas de diferentes materiales e incluso combinaciones de materiales y pueden reutilizarse. Han sido poco evaluadas y existe variabilidad en la filtración que dependerá del tipo de material, diferentes combinaciones de materiales, número de capas, forma, recubrimiento y mantenimiento. La disminución en la filtración es 2.5 veces mayor en mascarillas fabricadas con más de una capa de materiales como nylon y poliéster $100 \%$, lo cual no sucede con materiales porosos como la gasa, con los cuales la eficacia en la filtración disminuye hasta un $3 \%$. Entre mayor sea el número de capas, mayor será la protección; sin embargo, ningu- 
na mascarilla casera protege contra aerosoles. Para el aseo de los de este tipo, se recomienda el lavado con agua a $60{ }^{\circ} \mathrm{C}$ y jabón o detergente. De preferencia, se debe seguir las instrucciones de lavado si es que se cuenta con ellas. ${ }^{33}$

En la revisión sistemática y metaanálisis realizado por Chu y colaboradores, que incluyó estudios sobre SARS, MERS y SARS-CoV-2, se encontró que el uso de mascarillas y respiradores tanto en la comunidad como en el medio hospitalario, aunado al distanciamiento social de al menos un metro de distancia, se asociaron con una reducción significativa del riesgo de infección. Se estudiaron mascarillas reutilizables de materiales como algodón (12-16 capas o gasa) y en el metaanálisis realizado hubo evidencia, aunque con baja certeza, de que los respiradores podrían proporcionar mejor protección que las mascarillas. ${ }^{34}$

Un estudio realizado por Asadi y su equipo evaluó la eficacia de diferentes tipos de mascarillas: de uso médico (quirúrgicas); respiradores KN95 (HB2626-2006 de acuerdo a la regulación en China); mascarillas caseras de papel; algodón una capa; algodón doble capa y respirador N95 (NIOSH), en la reducción de la emisión de partículas de aerosoles analizadas por un medidor aerodinámico de partículas entre 0.3 a $20 \mu \mathrm{m}$ en personas sanas, realizando cuatro actividades principales: respirar, hablar, toser y masticación. Las mascarillas quirúrgicas y respiradores (KN95) redujeron significativamente la tasa de partículas emitidas hasta en 90 y $75 \%$, comparadas con no usar alguna mascarilla, en especial durante el habla y la tos respectivamente. La tasa de emisión de partículas de aerosoles, a través de mascarillas caseras de una sola capa de algodón, resultó considerablemente mayor a la observada al no usar mascarilla durante la respiración y el habla, mientras que al comparar las mascarillas de doble capa de algodón con no usar una mascarilla, no se encontró diferencia estadísticamente significativa. Lo anterior fue atribuido a la fricción y emisión de partículas de material (algodón) de tamaño similar a partículas de aerosoles emitidas durante las actividades realizadas, sin que esto tradujera necesariamente la emisión de partículas de virus, ya que sólo se midieron el número y tamaño de partículas emitidas. ${ }^{35}$

\section{Recomendaciones sobre el uso de cubrebocas}

De acuerdo con el tipo de actividades realizadas, el uso de cubrebocas o mascarillas faciales puede resumirse de la siguiente forma:
- Uso hospitalario: todas aquellas personas que laboren en una unidad de atención médica, sin importar las actividades realizadas.

En ausencia de actividades que impliquen la generación de aerosoles, la Organización Mundial de la Salud recomienda el uso de mascarillas quirúrgicas.

En procedimientos que generen aerosoles, la recomendación es el uso de respiradores (N95, FFP2 o FFP3 o equivalente). Asimismo, el uso de estos respiradores es indispensable en la atención directa de pacientes con COVID-19. En las áreas clínicas en donde el personal brinde atención directa a pacientes, independientemente del diagnóstico de COVID-19, se recomienda el uso de un cubrebocas o mascarilla quirúrgica durante todo el turno y desecharla al terminar la atención del paciente si esto implicó la generación de gotas, o bien, cuando está húmeda o presenta alguna alteración en su integridad, con la finalidad de evitar contaminación cruzada.

- Uso en la comunidad: hasta el momento, se han realizado recomendaciones sobre el uso de mascarillas en la comunidad en diferentes países con la finalidad de suprimir la transmisión de SARS-CoV-2, teniendo como propósitos: evitar la transmisión del virus de una persona infectada, ofrecer protección a personas sanas, evitar el riesgo de exposición, evitar la exposición de personas vulnerables como pacientes inmunocomprometidos y con comorbilidades asociadas. Para que la protección sea más efectiva, se requiere el uso de mascarillas de uso no médico para la población general, y el uso de mascarillas quirúrgicas para la población vulnerable. ${ }^{36}$

\section{Recomendaciones sobre del uso de mascarillas en niños}

De acuerdo a la evidencia actual, los niños parecen tener menor susceptibilidad a la infección por SARSCoV-2 comparados con los adultos; sin embargo, los datos hasta ahora demuestran que esto puede variar con la edad.

En cuanto al uso de mascarillas en niños, algunos estudios han evaluado la eficacia en brotes de influenza y otros virus respiratorios. Una investigación realizada durante brotes de influenza en Japón reportó que el uso 
de mascarillas fue más efectivo en niños escolares (9-12 años de edad) comparado con niños más pequeños. ${ }^{37}$ Aún no hay publicaciones que evalúen el impacto de las mascarillas durante el juego y actividad física en niños. Existen pocos estudios que evalúen la eficacia del uso de mascarillas en niños, algunos de ellos reportan una menor eficacia probablemente atribuida al tamaño de las mascarillas y pobre ajuste a la cara de los niños pequeños.

La Organización Mundial de la Salud y ante la ausencia de estudios en niños, sugieren seguir las siguientes recomendaciones de expertos: ${ }^{38}$

- El uso de mascarillas será para mayores de cinco años, explicado por la adquisición de hitos del desarrollo, que permitan el uso correcto de las mismas con la mínima ayuda requerida por un adulto y la mínima manipulación de la mascarilla por parte del niño.

- Los niños con problemas cognitivos o respiratorios con dificultad para tolerar la mascarilla no deben utilizarla.

- El uso de mascarillas en niños de seis a 11 años deberá realizarse evaluando la capacidad del niño para la colocación correcta de la mascarilla y la capacidad de supervisión de un adulto.

- Los niños mayores de 12 años deberán seguir las recomendaciones para adultos.

- Como consideraciones especiales para niños con retraso en el neurodesarrollo, problemas auditivos o dificultad en el aprendizaje, en los que el uso de mascarillas podría condicionar mayor dificultad en el aprendizaje, se debe evaluar el riesgo y deberán ofrecerse alternativas como caretas o mascarillas transparentes.

\section{Recomendaciones en cuanto al uso de caretas en situaciones especiales}

Al no ser una barrera física completa, ésta debe cubrir la cara por completo hasta por debajo del mentón. Para reutilizarse, deberán ser lavadas con jabón o detergente y agua, desinfectadas con alcohol al 7090\% y guardadas después de su uso. Además deberá mantenerse el distanciamiento físico de al menos un metro. $^{36}$

\section{CONCLUSIONES}

La situación actual de la pandemia por COVID-19, con cada vez más casos y muertes en el mundo y debido a su comportamiento epidémico, ha generado un incremento en la evidencia científica con respecto a la enfermedad. $\mathrm{Al}$ momento no se cuenta con un tratamiento dirigido, por esta razón, la prioridad es continuar con las medidas de prevención de acuerdo a las recomendaciones internacionales.

El uso de mascarillas es la medida preventiva principal para evitar la propagación de COVID-19, por lo cual es prioritario seguir promoviendo el uso de éstas, tanto en la población general como en el medio hospitalario, con la finalidad de lograr contener la pandemia.

Debe recomendarse su uso en la comunidad independientemente del material utilizado, prefiriéndose en el caso de las caseras que sean de al menos dos capas de material no poroso. Las mascarillas de uso médico (mascarillas quirúrgicas, N95 y KN95) deben reservarse para su uso en medio hospitalario y, en la comunidad, solamente para pacientes vulnerables. El uso de mascarillas por sí solo no es eficaz para controlar la pandemia y por lo anterior deberán continuarse las demás medidas de prevención, como el lavado de manos y distanciamiento social, que en conjunto podrán contribuir a contener la pandemia mientras no se cuente con vacunación efectiva, así como un tratamiento específico eficaz.

\section{REFERENCIAS}

1. World Health Organization. Coronavirus disease (COVID-19). Weekly Epidemiological Update, Available in: https://www.who. int/docs/default-source/coronaviruse/situation-reports/20201012weekly-epi-update-9.pdf.

2. Hui DS, I Azhar E, Madani TA, Ntoumi F, Kock R, Dar O et al. The continuing 2019-nCoV epidemic threat of novel coronaviruses to global health - The latest 2019 novel coronavirus outbreak in Wuhan, China. Int J Infect Dis. 2020; 91: 264-266. doi: 10.1016/j. ijid.2020.01.009.

3. Cui J, Li F, Shi ZL. Origin and evolution of pathogenic coronaviruses. Nat Rev Microbiol. 2019; 17(3): 181-192. doi: 10.1038/s41579-018-0118-9.

4. Jiang S, Du L, Shi Z. An emerging coronavirus causing pneumonia outbreak in Wuhan, China: calling for developing therapeutic and prophylactic strategies. Emerg Microbes Infect. 2020; 9(1): 275277. doi: $10.1080 / 22221751.2020 .1723441$.

5. Chan JF, Yuan S, Kok KH, To KK, Chu H, Yang J et al. A familial cluster of pneumonia associated with the 2019 novel coronavirus indicating person-to-person transmission: a study of a family cluster. Lancet. 2020; 395(10223): 514-523. doi: 10.1016/S01406736(20)30154-9.

6. Chen N, Zhou M, Dong X, Qu J, Gong F, Han Y, Qiu Y, Wang J et al. Epidemiological and clinical characteristics of 99 cases of 2019 novel coronavirus pneumonia in Wuhan, China: a descriptive study. Lancet. 2020; 395(10223): 507-513. doi: 10.1016/S01406736(20)30211-7 
7. Gralinski LE, Menachery VD. Return of the coronavirus: 2019nCoV. Viruses. 2020; 12(2): 135. doi: 10.3390/v12020135.

8. Gorbaleny AE, Baker SC, Baric RS, De Groot RJ, Drosten C, Gulyaeva $\mathrm{C}$ et al. The species Severe acute respiratory syndromerelated coronavirus: classifying $2019-\mathrm{nCoV}$ and naming it SARSCoV-2. Nat Microbiol. 2020; 5: 536-544. doi: org/10.1038/s41564020-0695-z.

9. Huang C, Wang Y, Li X, Ren L, Zhao J, Hu Y et al. Clinical features of patients infected with 2019 novel coronavirus in Wuhan, China. Lancet. 2020; 395(10223): 497-506.

10. Wang D, Hu B, Hu C, Zhu F, Liu X, Zhang J et al. Clinical Characteristics of 138 Hospitalized Patients With 2019 Novel Coronavirus-Infected Pneumonia in Wuhan, China. JAMA. 2020; 323(11): 1061-1069. doi: 10.1001/jama.2020.1585.

11. Guan WJ, Ni ZY, Hu Y, Liang WH, Ou CQ, He JX et al; China Medical Treatment Expert Group for COVID-19. Clinical characteristics of coronavirus disease 2019 in China. N Engl J Med. 2020; 382(18): 1708-1720. doi: 10.1056/ NEJMoa2002032.

12. Lu X, Zhang L, Du H, Zhang J, Li YY, Qu J; Chinese Pediatric Novel Coronavirus Study Team. SARS-CoV-2 Infection in Children. N Engl J Med. 2020; 382(17): 1663-1665. doi: 10.1056/ NEJMc2005073.

13. Liguoro I, Pilotto C, Bonanni M, Ferrari ME, Pusiol A, Nocerino A et al. SARS-COV-2 infection in children and newborns: a systematic review. Eur J Pediatr. 2020; 179(7): 1029-1046. doi: 10.1007/ s00431-020-03684-7.

14. Cheng VC, Wong SC, Chen JH, Yip CC, Chuang VW, Tsang OT et al. Escalating infection control response to the rapidly evolving epidemiology of the coronavirus disease 2019 (COVID-19) due to SARS-CoV-2 in Hong Kong. Infect Control Hosp Epidemiol. 2020; 41(5): 493-498.

15. Ong SW, Tan YK, Chia PY, Lee TH, Ng OT, Wong MS et al. Air, surface environmental, and personal protective equipment contamination by severe acute respiratory syndrome coronavirus 2 (SARS-CoV-2) from a symptomatic patient. JAMA. 2020; 323(16): 1610-1612. doi:10.1001/jama.2020.3227.

16. Centers for Disease Control and Prevention. Coronavirus Disease 2019 (COVID-19). Appendices. [Access 25 October 2020] Available in: https://www.cdc.gov/coronavirus/2019ncov/php/contact-tracing/contact-tracing-plan/appendix. html\#contact.

17. Siegel JD, Rhinehart E, Jackson M, Chiarello L; the Healthcare Infection Control Practices Advisory Committee. 2007 Guidelines for isolation precautions: preventing transmission of infectious agents in healthcare settings. Am J Infect Control. 2007; 35(Suppl 2): S65-S164

18. Jones RM, Brosseau LM. Aerosol transmission of infectious disease. J Occup Environ Med. 2015; 57(5): 501-508. doi: 10.1097/ JOM.0000000000000448.

19. Johnson GR, Morawksa L, Ristovski ZD, Hargreaves M, Mengersen K, Chao CY et al. Modality of human expired aerosol size distributions. J Aerosol Sci. 2011; 42: 839-851.

20. Van Doremalen N, Bushmaker T, Morris DH, Holbrook MG Gamble A, Williamson BN et al. Aerosol and Surface Stability of SARS-CoV-2 as Compared with SARS-CoV-1. N Engl J Med. 2020; 382(16): 1564-1567.

21. Fears A, Klimstra W, Duprex P, Hartman A, Weaver SC, Plante KS et al. Comparative dynamic aerosol efficiencies of three emergent coronaviruses and the unusual persistence of SARS-CoV-2 in aerosol suspensions. MedRxiv. [preprint]. [Access 22 October 2020] Available in: https://www.medrxiv.org/content/10.1101/20 20.04.13.20063784v1.full.pdf+html.
22. Yu P, Zhu J, Zhang Z, Han Y. A Familial Cluster of Infection associated with the 2019 novel coronavirus indicating possible person-to-person transmission during the incubation period. $J$ Infect Dis. 2020; 221(11): 1757-1761.

23. Lauer SA, Grantz KH, Bi Q, Jones FK, Zheng Q, Meredith HR et al. The incubation period of coronavirus disease 2019 (COVID-19) from publicly reported confirmed cases: estimation and application. Ann Intern Med. 2020; 172(9): 577-582.

24. Li R, Pei S, Chen B, Song Y, Zhang T, Yang W, Shaman J. Substantial undocumented infection facilitates the rapid dissemination of novel coronavirus (SARS-CoV-2). Science. 2020; 368(6490): 489-493. doi: 10.1126/science.abb3221.

25. Canini L, Andreoletti L, Ferrari P, D'Angelo R, Blanchon T, Lemaitre $\mathrm{M}$ et al. Surgical mask to prevent influenza transmission in households: a cluster randomized trial. PLoS One. 2010; 5(11): e13998.

26. MacIntyre CR, Zhang Y, Chughtai AA, Seale H, Zhang D, Chu Y et al. Cluster randomised controlled trial to examine medical mask use as source control for people with respiratory illness. BMJ Open. 2016; 6(12): e012330.

27. European Standards. UNE EN 14683:2019+AC:2019. Medical Face Masks -Requirements and Test Methods. 2019. [Access 23 October 2020] Available in: https://www.en-standard.eu/une-en14683-2019-ac- 2019-medical-face-masks-requirements-and-testmethods/.

28. F23 Committee, n.d. Specification for Performance of Materials Used in Medical Face Masks. ASTM International. Available in: https://doi.org/10.1520/F2100-19E01 (Access 9 October 2020).

29. National Institute for Occupational Safety and Health (NIOSH). $\mathrm{NIOSH}$ Guide to the Selection and Use of Particulate Respirators. Department of Health and Human Services (DHHS) NIOSH publication number 96- 101, 1996. [Access 20 October 2020] Available in: http://www.cdc.gov/niosh/userguid.html.

30. Centers for Disease Control and Prevention. Considerations for Wearing Masks. Help Slow de Spread of COVID-19. [Access 23 October 2020] Available in: https://www.cdc.gov/coronavirus/2019ncov/prevent-getting-sick/cloth-face-cover-guidance.html.

31. Centers for Disease Control and Prevention. Coronavirus Disease 2019 (COVID-19). Personal Protective Equipment FAQs. [Access 23 October 2020] Available in: https://www.cdc.gov/ coronavirus/2019-ncov/hcp/respirator-use-faq.html.

32. Verma S, Dhanak M, Frankenfield J. Visualizing droplet dispersal for face shields and masks with exhalation valves. Phys Fluids. 2020; 32(9): 091701. doi: 10.1063/5.0022968.

33. Jung H, Kim JK, Lee S, Lee J, Kim J, Tsai P et al. Comparison of filtration efficiency and pressure drop in anti-yellow sand masks, quarantine masks, medical masks, general masks, and handkerchiefs. Aerosol Air Qual Res. 2014;14, 991-1002. doi. org/10.4209/aaqr.2013.06.0201.

34. Chu DK, Akl EA, Duda S, Solo K, Yaacoub S, Schünemann HJ. COVID-19 systematic urgent review group effort (SURGE) study authors. Physical distancing, face masks, and eye protection to prevent person-to-person transmission of SARSCoV-2 and COVID-19: a systematic review and meta-analysis. Lancet. 2020; 395 (10242): 1973-1987. doi: 10.1016/S01406736(20)31142-9.

35. Asadi S, Cappa CD, Barreda S, Wexler AS, Bouvier NM, Ristenpart WD. Efficacy of masks and face coverings in controlling outward aerosol particle emission from expiratory activities. Sci Rep. 2020; 10: 15665 . doi:10.1038/s41598-020-72798-7.

36. World Health Organization. Advice on the use of masks in the context of COVID-19. [Access 23 October 2020] Available in: https://apps.who.int/iris/bitstream/ 
handle/10665/332293/WHO-2019-nCov-IPC Masks-2020.4eng.pdf?sequence=1\&isAllowed $=y$.

37. Uchida M, Kaneko M, Hidaka Y, Yamamoto H, Honda T, Takeuchi $S$ et al. Effectiveness of vaccination and wearing masks on seasonal influenza in Matsumoto City, Japan, in the 2014/2015 season: an observational study among all elementary schoolchildren. Prev Med Rep. 2017; 5: 86-91. Epub 2016/12/17.

38. World Health Organization. Advice on the use of masks for children in the community in the context of COVID-19. Available in: https:// www.who.int/publications/i/item/WHO-2019-nCoV-IPC_MasksChildren-2020.1. 